\title{
Diagnostic values of red cell distribution width, platelet distribution width and neutrophil-lymphocyte count ratio for sepsis
}

\author{
HUI-BING ZHANG, JUAN CHEN, QIAO-FEN LAN, XIONG-JIAN MA and SHI-YAN ZHANG \\ Clinical Laboratory, Affiliated Fuding Hospital, Fujian University of Traditional Chinese Medicine, \\ Fuding, Fujian 355200, P.R. China
}

Received May 19, 2015; Accepted July 5, 2016

DOI: $10.3892 /$ etm.2016.3583

\begin{abstract}
The aim of this study was to evaluate the diagnostic efficiency of red blood cell distribution width (RDW), platelet distribution width (PDW), the neutrophil-lymphocyte count ratio (NLCR), procalcitonin (PCT) and $\mathrm{C}$-reactive protein (CRP) for the prediction of sepsis. A total of 120 consecutive patients who underwent blood culture testing were included. The PCT and CRP levels, and RDW, PDW and NLCR percentages were determined and compared between patients with positive blood cultures and those without. The PCT, CRP, RDW, PDW and NLCR values were significantly higher in patients with positive blood culture compared with those without. PCT and NLCR each had a high diagnostic performance for the prediction of sepsis, with an area under the curve (AUC) for sepsis of 0.829 and 0.718 , respectively. A combination of RDW, PDW and NLCR also exhibited a good diagnostic performance for sepsis (AUC, 0.704). NLCR is easily obtained by automated hematological analysis. Moreover, NLCR was found to have a high diagnostic efficiency for the prediction of sepsis, with greater sensitivity and accuracy than CRP. In conclusion, PCT exhibited the optimal diagnostic performance among the tested markers. The combination of the three parameters
\end{abstract}

Correspondence to: Dr Shi-Yan Zhang, Clinical Laboratory, Affiliated Fuding Hospital, Fujian University of Traditional Chinese Medicine, 120 South Road of Old City, Fuding, Fujian 355200, P.R China

E-mail: myebox@139.com

Abbreviations: RDW, red blood cell distribution width; PDW, platelet distribution width; NLCR, neutrophil-lymphocyte count ratio; PCT, procalcitonin; CRP, C-reactive protein; ROC, receiver operating characteristic; AUC, area under ROC curve; BSI, bloodstream infection; SIRS, systemic inflammatory response syndrome; PPV, positive predictive value; NPV, negative predictive value; $\mathrm{CI}$, confidence interval; $\mathrm{SE}$, standard error

Key words: red cell distribution width, platelet distribution width, neutrophil-to-lymphocyte ratio, procalcitonin, C-reactive protein, ROC curve of RDW, PDW and NLCR, demonstrated a high diagnostic performance similar to that of PCT.

\section{Introduction}

Sepsis is a bloodstream infection (BSI) that is typically associated with severe, whereas bacteremia is the presence of viable bacteria in the bloodstream and, as it may cause no symptoms, may resolve without treatment. In some cases, bacteremia may lead to sepsis. Blood culture has been commonly used as a traditional approach for the detection of sepsis. However, blood cultures are time-consuming and at least 2-5 days of culture is necessary to identify the bacterial or fungal organism in the blood. Moreover, the sensitivity of blood cultures declines significantly if antibiotic therapy has been initiated, or when fastidious or slow-growing pathogens are cultured (1). Therefore, other types of test, such as a complete blood count and a chemistry panel are ordered along with or prior to the blood culture to determine whether the patient has a BSI.

The red blood cell distribution width (RDW) and platelet distribution width (PDW) are parts of a routine complete blood count. RDW is a means of evaluating the variability in size of erythrocytes and has been used widely in the differential diagnosis of anemia (2). PDW is measure of variation in platelet size, which may be an indicator of active platelet release (3). A number of previous investigations have shown that RDW and PDW are significantly associated diseases including chronic spontaneous urticaria and gram-negative bacteremia (3-5). Furthermore, RDW has also been regarded as a prognostic marker for certain diseases, such as stable angina pectoris $(6,7)$.

Zahorec first proposed the use of the ratio of neutrophil and lymphocyte counts [neutrophil lymphocyte count ratio (NLCR)] as an marker of infection in clinical applications (8). Recently, an investigation by Loonen et al (1) demonstrated that NLCR was a rapidly available biomarker, which appeared most promising in differentiating patients with BSI from those without BSI for subsequent pathogen identification (1). NLCR has been suggested to be a better predictor of bacteremia than routine parameters such as C-reactive protein (CRP) levels, white blood cell count and neutrophil count (9). 
CRP is an acute-phase protein mostly produced by the liver following the onset of inflammation. Procalcitonin (PCT) is normally produced by C-cells in the thyroid gland as a precursor to calcitonin (10). It is present in low levels in the blood of healthy subjects. Recently, CRP and PCT have been investigated for potential to serve as important tools for the early diagnosis of bacterial infection in clinical applications $(11,12)$.

In the present study, the RDW, PDW and NLCR parameters, which are parts of a complete blood count, were compared with the traditional parameters CRP and PCT for ability to predict sepsis in patients with or without positive blood cultures.

\section{Materials and methods}

Patients. The clinical and laboratory data of 120 consecutive patients who were tested by blood culture at the Affiliated Fuding Hospital of Fujian University of Traditional Chinese Medicine (Fuding, China) between January 2014 and March 2015 were retrospectively analyzed. Clinical and laboratory data were regarded as eligible when a patient met the following inclusion criteria: i) The presence of two or more diagnostic criteria for systemic inflammatory response syndrome (SIRS) and suspected or documented infection (13); ii) all blood samples of the studied patient were collected simultaneously during the same septic episode (14). The exclusion criteria were as follows: i) Patients with hematological diseases such as hematological malignancy, metastatic bone marrow infiltration by malignancy, recovery after bone marrow hyperplasia or acute bleeding (15); ii) absence of at least two SIRS diagnostic criteria (13); iii) samples not collected simultaneously from the same studied patient. The patients were classified into two groups according to the results of the blood culture. Patients with a positive blood culture constituted the positive group and those with a negative blood culture were classified into the negative group. There were 33 males and 27 females with a mean age of $62.6 \pm 14.9$ years (range, $21-89$ years) in the positive group, and 40 males and 20 females with a mean age of 66.0 \pm 15.3 years (range, 24-90 years) in the negative group. The protocol was approved by the ethics committee of the Affiliated Fuding Hospital of Fujian University of Traditional Chinese Medicine.

Blood samples were extracted from peripheral venous puncture of the patients, directly injected into Bactec bottles, and incubated in a Bactec incubator (BD Diagnostics, Franklin Lakes, NJ, USA). Blood culture bottles were incubated for 7 days at $37^{\circ} \mathrm{C}$. Positive cultures were Gram-stained, and subcultured on solid media for subsequent analysis. Identification of microorganisms was conducted with conventional techniques and with an API system (bioMérieux, Marcy-l'Étoile, France).

Marker determination. CRP analysis was performed using the Dimension Vista 1500 Intelligent Lab system (Siemens Healthcare GmbH, Erlangen, Germany) according to the manufacturer's protocol. Complete blood cell counts and mean corpuscular volume (MCV) were determined using a Sysmex XE-2100 hematology analyzer (Sysmex Corporation, Kobe, Japan). The NLCR was determined by dividing the absolute neutrophil count by the absolute lymphocyte count, and PCT levels were measured using a Cobas E411 analyzer (Roche Diagnostics, Basel, Switzerland).

Statistical analysis. Categorical and continuous variables were expressed as frequency, mean \pm standard deviation, or median and interquartile range. Testing of data for normal distribution was carried out by means of the Kolmogorov-Smirmov test. Comparison of continuous variables between the two groups was performed using the Mann-Whitney U test. Receiver operating characteristic (ROC) curve analyses were conducted for the single markers and the combination RDW + PDW + NLCR in the prediction of blood culture positivity. The area under the ROC curve (AUC) was calculated for each marker, and the standard error and $95 \%$ confidence interval $(95 \% \mathrm{CI})$ were determined. The sensitivity, specificity, accuracy, positive predictive value (PPV) and negative predictive value (NPV) were calculated in accordance with the ROC curves. In all tests, $\mathrm{P}<0.05$ was considered to indicate a statistically significant difference. Statistical analysis was performed using the SPSS version 22.0 software package (IBM SPSS, Armonk, NY, USA).

\section{Results}

Study population. The current study was performed on a total of 120 patients who underwent blood culture tests in hospital. The major attributes of the patients in the study are presented in Table I. Statistical analysis demonstrated that there was no significant difference with respect to age or gender.

Kolmogorov-Smirmov test. The statistical distribution of every tested marker in each group was analyzed by means of a Kolmogorov-Smirmov test. The results were $\mathrm{Z}=0.123-0.407$, $\mathrm{P}<0.01$, indicating non-normal distribution, and non-parametric tests were conducted in the following statistical analysis.

Levels of markers in the two groups. The concentrations of PCT and CRP, and the RDW, PDW and NLCR values were significantly higher in the positive group compared with those in negative group. However, no significant difference in MCV was observed between the two groups (Table II).

Evaluation of markers and ROC analysis. The sensitivity, specificity, Youden's index (sensitivity + specificity - 1) and diagnostic accuracy of PCT, CRP, RDW, PDW, NLCR and the combination RDW + PDW + NLCR were calculated on the basis of the ROC curves and logistic regression (Table III). Among the tested markers, PCT had the highest sensitivity $(91.70 \%)$ and accuracy $(80.60 \%)$ and the PDW had the highest specificity $(84.70 \%)$ for predicting sepsis.

The ROC curves for the analysis of markers in patients with positive blood culture compared with those in patients without positive blood culture were plotted. The AUCs of the markers are shown in Table IV. The largest AUC was 0.829 (95\% CI: 0.754-0.905) for PCT, while the AUC was 0.666 (95\% CI: 0.568-0.764) for CRP. The ROC analysis, conducted to evaluate the diagnostic accuracy along with the specificity, sensitivity, PPV and NPV of the optimal cut-off values of the different markers in the prediction of sepsis are shown in 
Table I. Major attributes of the two groups.

\begin{tabular}{|c|c|c|c|c|c|}
\hline Attribute & Positive group $(n=60)$ & Negative group $(n=60)$ & Student's t-test & $\chi^{2}$ & P-value \\
\hline Age (mean $\pm \mathrm{SD}$, years $)$ & $62.6 \pm 14.9$ & $66.0 \pm 15.3$ & -1.234 & & 0.220 \\
\hline Gender, n (\%) & & & & 1.259 & 0.190 \\
\hline Male & $33(55.0)$ & $40(66.7)$ & & & \\
\hline Female & $27(45.0)$ & $20(33.3)$ & & & \\
\hline
\end{tabular}

SD, standard deviation.

Table II. Mean results for markers in the two groups [median $\left(\mathrm{P}_{25}-\mathrm{P}_{75}\right)$ ].

\begin{tabular}{|c|c|c|c|c|c|c|}
\hline Group & PCT (ng/ml) & $\mathrm{CRP}(\mathrm{mg} / \mathrm{l})$ & $\operatorname{MCV}(\%)$ & RDW (\%) & PDW (\%) & $\operatorname{NLCR}(\%)$ \\
\hline Positive & $9.27(1.34-38.53)$ & $147.0(55.9-200.0)$ & $90.6(86.6-93.3)$ & $14.1(13.4-15.2)$ & $12.4(11.1-15.1)$ & $16.9(9.8-26.5)$ \\
\hline Negative & $0.18(0.05-0.86)$ & $55.9(19.7-119.2)$ & $91.6(88.2-94.4)$ & $13.6(12.7-14.7)$ & $11.6(10.6-12.6)$ & $8.38(4.4-16.0)$ \\
\hline Z-value & -6.270 & -3.586 & -3.586 & -2.318 & -2.544 & -4.078 \\
\hline P-value & $<0.001$ & $<0.001$ & 0.274 & 0.020 & 0.011 & $<0.001$ \\
\hline
\end{tabular}

Mann-Whitney U-test was carried out to compare results between the two groups. P25, 25th percentile; P75, 75th percentile; PCT, procalcitonin; CRP, C-reactive protein; MCV, mean corpuscular volume; RDW, red blood cell distribution width; PDW, platelet distribution width; NLCR, neutrophil-lymphocyte count ratio.

Table III. Comparison of performance characteristics of individual markers and a marker combination in predicting sepsis.

\begin{tabular}{lccccccc}
\hline Markers & Cut off & Sensitivity $(\%)$ & Specificity $(\%)$ & Accuracy $(\%)$ & PPV (\%) & NPV (\%) & Youden's index (\%) \\
\hline PCT & 0.37 & 91.70 & 69.50 & 80.60 & 75.00 & 89.34 & 61.20 \\
CRP & 126.25 & 53.30 & 79.70 & 66.50 & 72.40 & 63.10 & 33.00 \\
RDW & 13.45 & 73.30 & 49.20 & 61.20 & 59.10 & 64.80 & 22.50 \\
PDW & 13.35 & 38.30 & 84.70 & 61.50 & 71.50 & 57.90 & 76.87 \\
NLCR & 9.37 & 81.10 & 62.70 & 71.92 & 68.51 & 73.80 & 75.80 \\
Combination & - & 80.00 & 62.70 & 71.40 & 68.20 & 42.70 \\
\hline
\end{tabular}

PPV, positive predictive value; NPV, negative predictive value; PCT, procalcitonin (ng/ml); CRP, C-reactive protein (mg/l); RDW, red blood cell distribution width (\%); PDW, platelet distribution width (\%); NLCR, neutrophil-lymphocyte count ratio (\%); combination, RDW + PDW + NLCR.

Fig. 1. PCT had the greatest accuracy, at the cut-off value of $0.37 \mathrm{ng} / \mathrm{ml}(91.70 \%$ sensitivity and $69.50 \%$ specificity). NLCR had the second best accuracy, at the cutoff value of $9.37 \%$ ( $81.10 \%$ sensitivity and $62.70 \%$ specificity).

\section{Discussion}

Sepsis and severe sepsis are major causes of morbidity and mortality (16). Early identification of the pathogen in sepsis plays a pivotal role in its adequate treatment, and it has been shown that when the appropriate treatment is initiated promptly the BSI-related mortality rate is reduced (17). By contrast, a delay in the diagnosis and treatment of sepsis will result in the rapid progression of circulatory failure, multiple organ dysfunction and eventually death (15). As a result, specific markers and molecular diagnostic assays (18) have been developed for rapid laboratory diagnosis in order to improve the clinical management of sepsis (14).

The present study concentrated on six markers that may be employed for the rapid diagnosis of sepsis. Consistent with findings of a previous study (14), PCT performed better than other markers of inflammation, with the highest accuracy being found at the cutoff of $0.37 \mathrm{ng} / \mathrm{ml}$, with a sensitivity of $91.70 \%$ and a specificity of $69.50 \%$. Likewise, in a study of 571 patients, Leli et al (14) found that predicting bacteremia in patients with suspected sepsis using the optimal cutoff value of $>0.5 \mathrm{ng} / \mathrm{ml}$ for PCT resulted in a sensitivity and specificity of 94 and 64\%, respectively. Furthermore, the present study found the largest AUC was 0.829 (95\% CI: 0.754-0.905) for PCT, while the AUC was 0.666 (95\% CI: 0.568-0.764 ) for CRP. These findings are in agreement with a previous investigation by Müller et al (19), which found 
Table IV. Area under the ROC curve of each tested marker.

$95 \%$ confidence interval

\begin{tabular}{lccccc} 
Markers & AUC & SE & P-value & Lower bound & Upper bound \\
\hline PCT & 0.829 & 0.039 & $<0.001$ & 0.754 & 0.905 \\
CRP & 0.666 & 0.050 & 0.002 & 0.568 & 0.764 \\
PDW & 0.636 & 0.051 & 0.010 & 0.537 & 0.741 \\
RDW & 0.621 & 0.051 & 0.023 & 0.520 & 0.722 \\
NLRC & 0.718 & 0.047 & $<0.001$ & 0.625 & 0.811 \\
Combination & 0.704 & 0.047 & $<0.001$ & 0.612 & 0.796 \\
\hline
\end{tabular}

AUC, area under the receiver operating characteristic curve; SE, standard error; PCT, procalcitonin;. CRP, C-reactive protein; RDW, red blood cell distribution width; PDW, platelet distribution width; NLCR, neutrophil-lymphocyte count ratio; combination, PDW + RDW + NLRC.

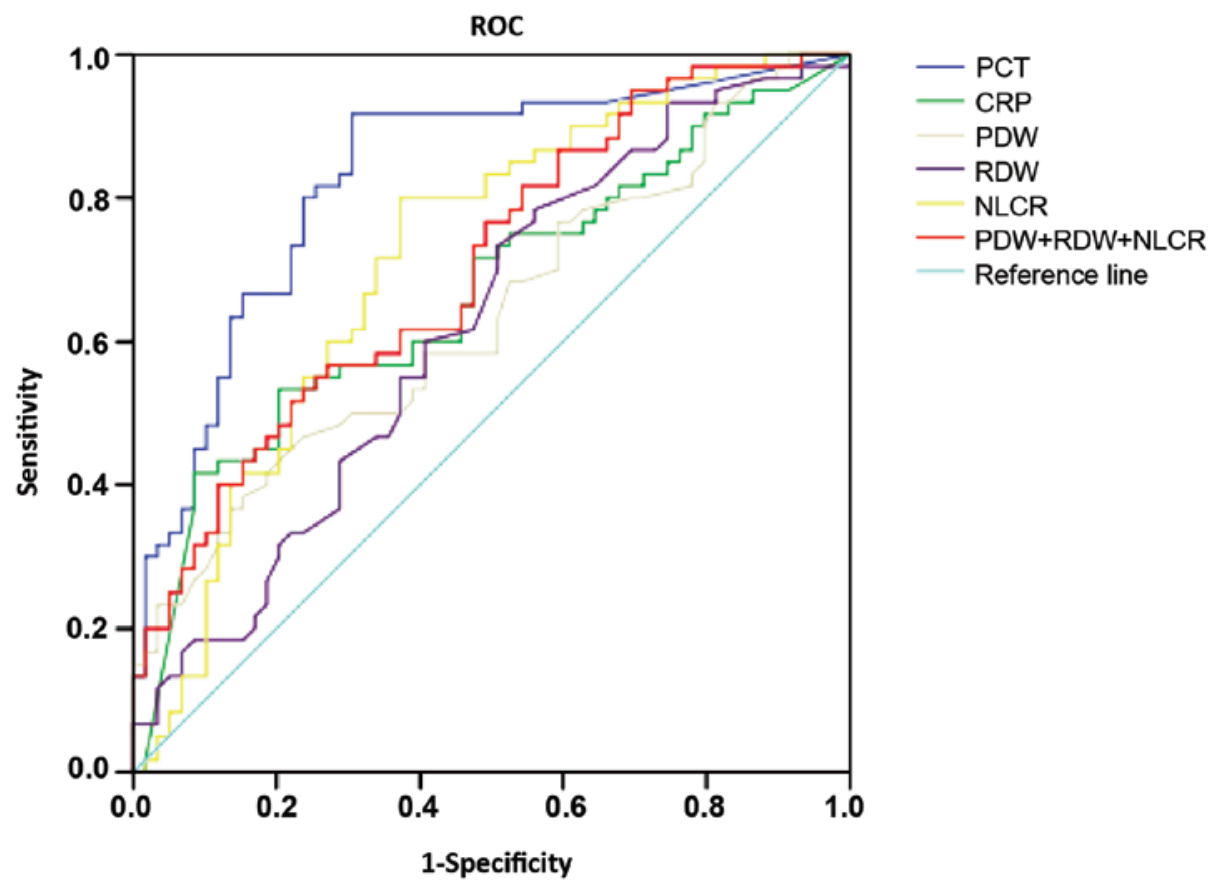

Figure 1. Receiver operating characteristic (ROC) curves comparing the ability of RDW, PDW, NLCR, PCT, CRP and the combination of three tested parameters (RDW + PDW + NLCR) to distinguish bacteremia from nonbacteremia. RDW, red blood cell distribution width; PDW, platelet distribution width; NLCR, neutrophil-lymphocyte count ratio; PCT, procalcitonin; CRP, C-reactive protein.

AUCs of 0.82 (95\% CI: 0.77-0.86) for PCT and 0.67 (95\% CI: 0.59-0.74) for CRP.

PDW is an index of platelet size heterogeneity and has been shown to be a marker of platelet function and activation (3). RDW is a measure of the variation of red blood cell size or red blood cell volume and is used in the differentiation of anemia (20). PDW and RDW can be obtained automatically as parts of the complete blood count. In the present study, the area under the ROC curves of RDW, PDW and NLCR were 0.621 (95\% CI: 0.520-0.722), 0.636 (95\% CI: 0.537-0.741) and 0.718 (95\% CI: $0.625-0.811)$, respectively. These results are similar to those of previous studies. A study by Guclu et al (21) found that the AUC of PDW was 0.733 (95\% CI: 0.678-0.783) with a sensitivity of $59.31 \%$ and a specificity of $76.22 \%$. An investigation by $\mathrm{Ku}$ et al (4) observed that the AUC of RDW was 0.764 (95\% CI: 0.650-0.879). Therefore, the two indices
PDW and RDW have diagnostic power in the discrimination of bacteremia. The results indicate that the elevated PDW, RDW values observed at the onset of inflammation are significantly associated with the presence of bacteremia. In the present study it was found that the AUC of the NLCR ROC curve was the second largest among the tested markers. The NLCR has been demonstrated to be a marker of infection with discriminatory capacity in predicting bacteremia (9). In a previous study, NLCR was regarded as a better marker than PCT in predicting bacteremia in patients suspected of having sepsis (9). On the basis of the data in the present study, it may be suggested that the finding of an increased PDW, RDW or NLCR value should alert clinicians to initiate or to change antibiotic treatment. The present study also revealed that if RDW, PDW and NLCR are considered together, the combination of the three tested parameters of the complete blood 
count has a higher diagnostic power than each single marker alone for the prediction of bacteremia.

In conclusion, PCT showed the best diagnostic performance among the tested markers. The combination of the three parameters of the complete blood count, namely RDW, PDW and NLCR, exhibited a high diagnostic performance similar to that of procalcitonin. NLCR was found to have a high diagnostic efficiency for the prediction of sepsis, with a greater sensitivity and accuracy than CRP. The RDW, PDW and NLCR values are obtained easily using automated hematological analysis and are cost-efficient measures. These three markers are rapidly available, inexpensive and easy to determine. The combination of these three tested parameters could be considered as a marker to distinguish bacteremia from nonbacteremia.

\section{References}

1. Loonen AJ, de Jager CP, Tosserams J, Kusters R, Hilbink M, Wever PC and van den Brule AJ: Biomarkers and molecular analysis to improve bloodstream infection diagnostics in an emergency care unit. PLoS One 9: e87315, 2014.

2. Miyamoto K, Inai K, Takeuchi D, Shinohara T and Nakanishi T: Relationships among red cell distribution width, anemia and interleukin-6 in adult congenital heart disease. Circ J 79: 1100-1106, 2015.

3. Kasperska-Zajac A, Grzanka A, Jarzab J, Misiołek M, Wyszyńska-Chłap M, Kasperski J and Machura E: The association between platelet count and acute phase response in chronic spontaneous urticaria. Biomed Res Int 2014: 650913, 2014.

4. Ku NS, Kim HW, Oh HJ, Kim YC, Kim MH, Song JE, Oh DH, Ahn JY, Kim SB, Jeong SJ, et al: Red blood cell distribution width is an independent predictor of mortality in patients with gram-negative bacteremia. Shock 38: 123-127, 2012.

5. Celik A, Aydin N, Ozcirpici B, Saricicek E, Sezen H, Okumus M, Bozkurt S and Kilinc M: Elevated red blood cell distribution width and inflammation in printing workers. Med Sci Monit 19: 1001-1005, 2013.

6. Ren H, Hua Q, Quan M, Chen H, Hou H, Wang L, Liu R and Yang Z: Relationship between the red cell distribution width and the one-year outcomes in Chinese patients with stable angina pectoris. Intern Med 52: 1769-1774, 2013.

7. Allen LA, Felker GM, Mehra MR, Chiong JR, Dunlap SH, Ghali JK, Lenihan DJ, Oren RM, Wagoner LE, Schwartz TA and Adams KF Jr: Validation and potential mechanisms of red cell distribution width as a prognostic marker in heart failure. J Card Fail 16: 230-238, 2010.
8. Zahorec R: Ratio of neutrophil to lymphocyte counts-rapid and simple parameter of systemic inflammation and stress in critically ill. Bratisl Lek Listy 102: 5-14, 2001.

9. de Jager CP, van Wijk PT, Mathoera RB, de Jongh-Leuvenink J, van der Poll T and Wever PC: Lymphocytopenia and neutrophil-lymphocyte count ratio predict bacteremia better than conventional infection markers in an emergency care unit. Crit Care 14: R192, 2010.

10. Maruna P, Nedelníková K and Gürlich R: Physiology and genetics of procalcitonin. Physiol Res 49 (Suppl 1): S57-S61, 2000.

11. Nargis W, Ibrahim M and Ahamed BU: Procalcitonin versus C-reactive protein: Usefulness as biomarker of sepsis in ICU patient. Int J Crit Illn Inj Sci 4: 195-199, 2014.

12. Jiang L, Feng B, Gao D and Zhang Y: Plasma concentrations of copeptin, C-reactive protein and procalcitonin are positively correlated with APACHE II scores in patients with sepsis. J Int Med Res 43: 188-195, 2015.

13. Bone RC, Balk RA, Cerra FB, Dellinger RP, Fein AM, Knaus WA, Schein RM and Sibbald WJ; ACCP/SCCM Consensus Conference Committee; American College of Chest Physicians/Society of Critical Care Medicine: Definitions for sepsis and organ failure and guidelines for the use of innovative therapies in sepsis. Chest 136 (Suppl 5): e28, 2009.

14. Leli C, Cardaccia A, Ferranti M, Cesarini A, D'Alò F, Ferri C, Cenci E and Mencacci A: Procalcitonin better than C-reactive protein, erythrocyte sedimentation rate and white blood cell count in predicting DNAemia in patients with sepsis. Scand J Infect Dis 46: 745-752, 2014.

15. Kim H, Kim Y, Lee HK, Kim KH and Yeo CD: Comparison of the delta neutrophil index with procalcitonin and C-reactive protein in sepsis. Clin Lab 60: 2015-2021, 2014.

16. Mayr FB, Yende S and Angus DC. Epidemiology of severe sepsis. Virulence 5: 4-11, 2014.

17. Finfer S: The surviving sepsis campaign: Robust evaluation and high-quality primary research is still needed. Intensive Care Med 36: 187-189, 2010.

18. Reinhart K, Bauer M, Riedemann NC and Hartog CS: New approaches to sepsis: Molecular diagnostics and biomarkers. Clin Microbiol Rev 25: 609-634, 2012.

19. Müller F, Christ-Crain M, Bregenzer T, Krause M, Zimmerli W, Mueller B and Schuetz P; ProHOSP Study Group: Procalcitonin levels predict bacteremia in patients with community-acquired pneumonia: A prospective cohort trial. Chest 138: 121-129, 2010.

20. Kara H, Degirmenci S, Bayir A, Ak A, Akinci M, Dogru A, Akyurek F and Kayis SA: Red cell distribution width and neurological scoring systems in acute stroke patients. Neuropsychiatr Dis Treat 11: 733-739, 2015.

21. Guclu E, Durmaz Y and Karabay O: Effect of severe sepsis on platelet count and their indices. Afr Health Sci 13: 333-338, 2013. 